\title{
An Experimental Investigation on Partial Replacement of Sand with Bottom Ash with and without Superplastisizer
}

\author{
T. Purushotham Reddy ${ }^{1}$, Dr. K. Rajasekhar ${ }^{2}$ \\ ${ }^{1}$ P.G (M.Tech) Student, Dept. of Civil Engineering, Siddartha Educational Academy Group of Institutions/Integrated Campus, Tirupati \\ (Rural)/JNTUA Ananthapuramu, (India) \\ ${ }^{2}$ Professor, Dept. of Civil Engineering, Siddartha Educational Academy Group of Institutions/Integrated Campus, Tirupati (Rural)/JNTUA \\ Ananthapuramu, (India)
}

\begin{abstract}
Sustainability is one of the major themes of the future in our society. The construction industry alone is making use of millions of tones of natural river sand every year, which creates environmental imbalance (depletion of water table etc.).Hence, it is the time to go which consist of alternative materials (marginal materials or mineral admixtures) which can replace sand effectively; The various physical properties and mechanical strength properties studied consist of compressive strength, splitting tensile strength. And also durability properties studied consist of calorimetric chlorination technique. Similarly, to investigate the effect of use of bottom ash as partial replacement of fine aggregate using with super plasticizer at various percentages i.e.; $10 \%$, 20\% and $30 \%$ on concrete properties such as compressive strength, split tensile strength test and also durability test. Compressive strength of bottom ash concrete at the curing age of 28 days was almost adequate to the normal concrete. Splitting tensile strength of concrete improved at percentages of replacement of bottom ash.
\end{abstract}

Keywords: Crossed fused renal ectopia, Fusion anomalies of kidney, Malrotation and migration of ureteric bud, Aberrant renal vessels, Left L shaped kidney

\section{Introduction}

\subsection{General}

Economical development predominantly goes for diminishment of negative ecological effect came about by development industry which is the biggest shopper of characteristic assets. Over duration of time, waste administration has turned into a standout amongst the most unpredictable and testing issue on the planet which is influencing nature.

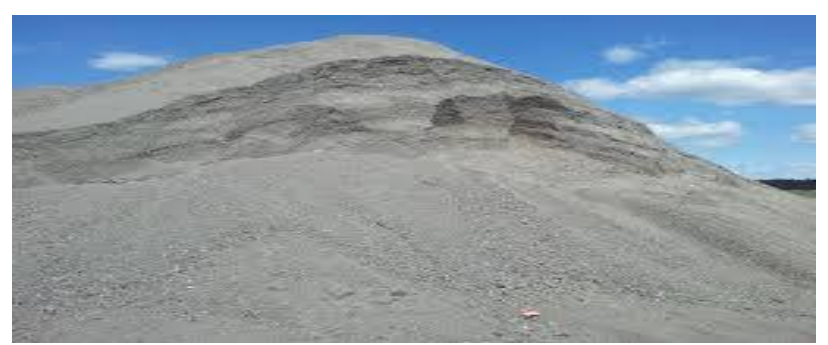

Figure 1.1: Bottom Ash

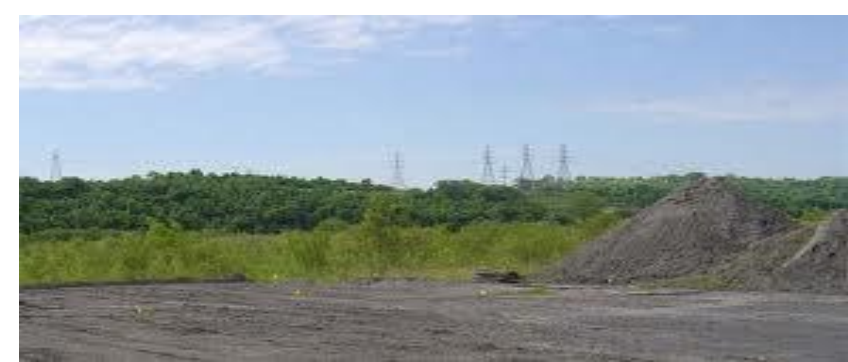

Figure 1.2: Rayalaseema Thermal Power Plant, Muddanur

The incombustible mineral components stick together until they are sufficiently substantial makes them expel from the base of the heaters either in a wet or dry condition and is transported to taking care of ranges by transport or funnel.

Bottom Ash is a skin and eye irritant, when it comes into contact.

- The bottom ash contains several toxic elements, such as lead $(\mathrm{Pb})$, zinc $(\mathrm{Zn})$, cadmium $(\mathrm{Cd})$ and copper $(\mathrm{Cu})$ can reach out and contaminate soils as well as surface water and groundwater.

- Bottom Ash was contact with water and pumping into artificial lagoon and landfill areas. This causes the pollution in water bodies and loss of productive land.

\subsection{Mineral Admixtures}

Mineral admixtures are usually added to concrete in larger amounts to enhance the workability of fresh concrete to improve resistance of concrete to thermal cracking, alkaliaggregate expansion, and sulphate attack and to enable a reduction in Cement content.

- Bottom ash

- Fly ash

- Silica Fume

- Ground Granulated Blast Furnace Slag

- Rice husk ash

Here, Bottom ash is used as partial replacement of sand.

\subsubsection{Bottom Ash}

Bottom Ash is coarse, with grain sizes spanning from sand to gravel, granular, incombustible by-product, and solid mineral residue. Bottom ash is formed when ash adheres shot particles to the boiler walls, agglomerates and then falls to the base of the furnace at a temperature around $12000 \mathrm{C}$. 


\section{International Journal of Science and Research (IJSR) \\ ISSN (Online): 2319-7064 \\ Index Copernicus Value (2013): 6.14 | Impact Factor (2015): 6.391}

Bottom ash is coarser than fly ash, with a sandy texture and particles ranging from about $0.1 \mathrm{~mm}$ to $50 \mathrm{~mm}$ in diameter.

\section{Factors Affecting the Utilization of bottom ash}

Some of the factors due to which, utilization of coal ash is not increasing at desired rate are

- Lack of awareness of coal ash properties.

- High transportation cost.

- Non-availability of dry fly ash and bottom ash

- Lack of proper co-ordination.

\subsubsection{Super plasticizer}

Modern concrete technology projections indicate a great expansion in low-cost and time efficient constructions. Concrete mix designs were likely more concerned on these chemical admixtures.

\subsubsection{VARAPLAST PC 432}

VARAPLAST PC 432 has been primarily developed for applications in the ready mixed and precast concrete industries where the highest durability and performance is required. Super plasticizing action of VARAPLAST PC 432isdifferent than conventional super plasticizers based on sulfonated melamine and naphthalene formaldehyde condensate, which create electrostatic repulsion of particles.

\subsection{Scope of the present work}

To achieve the aim of the project, necessary and essential on cement, fine aggregate, coarse aggregate, bottom ash and concrete of different mix need to be carried out.

\section{5 present work}

In the present study cement concrete cubes of $10 \%, 20 \%$ and $30 \%$ partial replacement of sand with BOTTOM ASH had been casted. The method adopted in this investigation used as per the IS code specifications is compression test to determine the strength adopted by the cubes for $7,14,28$, 56, 90 days.

Along with that another concrete cubes of 10\%, 20\% and $30 \%$ partial replacement of sand with BOTTOM ASH without super plasticizer with super plasticizer content as $4 \%$ constant for each proportionate mix had been casted and the compressive strength also tested as per IS code specification for $7,14,28,56,90$ days.

\section{Materials}

\subsubsection{Ordinary portland cement (53 grade)}

Cement may be described as a material with adhesive and cohesive properties that make it capable of bonding, mineral fragments into a compact whole. Most cement used today is Portland cement. This is carefully proportioned and specially processed combination of lime, silica, iron oxide and alumina

\subsubsection{Bottom Ash}

The Bottom Ash (BA) used for this study was obtained from Rayalaseema Thermal Power Plant (RTPP), Middalur, Kadapa (Dist). "Bottom Ash" is coarse, with grain sizes spanning from sand to gravel, granular, incombustible byproduct, and solid mineral residue. Bottom ash is formed when ash adheres a shot particles to the boiler walls, agglomerates and then falls to the base of the furnace at a temperature around $12000 \mathrm{C}$.

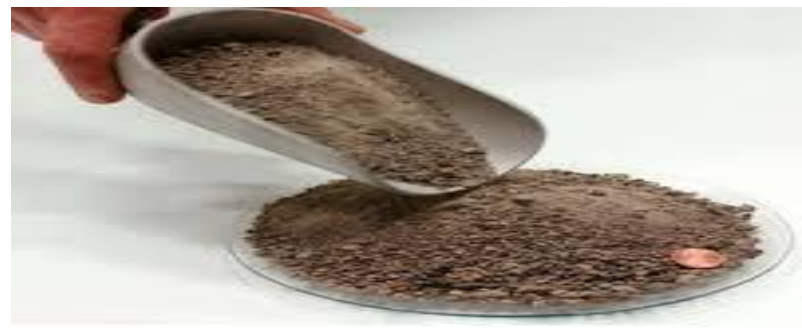

Figure 3.2: Bottom Ash

Table 3.11: Range of physical and chemical properties of bottom ash

\begin{tabular}{|c|c|c|}
\hline S. No & Characteristics & Test Result \\
\hline 1 & Colour & Grey to black in color \\
\hline 2 & Specific Gravity & 1.89 \\
\hline 3 & Bulk Density & $1130 \mathrm{~kg} / \mathrm{m} 3$ \\
\hline 4 & Fineness Modulus & 2.73 \\
\hline
\end{tabular}

Table 3.12: Chemical Composition of Bottom Ash

\begin{tabular}{|c|c|c|}
\hline S. No & Element & Mass \% \\
\hline 1 & $\mathrm{C}$ & 15.04 \\
\hline 2 & $\mathrm{O}$ & 60.27 \\
\hline 3 & $\mathrm{Al}$ & 9.02 \\
\hline 4 & $\mathrm{Si}$ & 12.62 \\
\hline 5 & $\mathrm{~K}$ & 0.64 \\
\hline 6 & $\mathrm{Ti}$ & 0.71 \\
\hline 7 & $\mathrm{Fe}$ & 1.69 \\
\hline
\end{tabular}

\subsection{Methods}

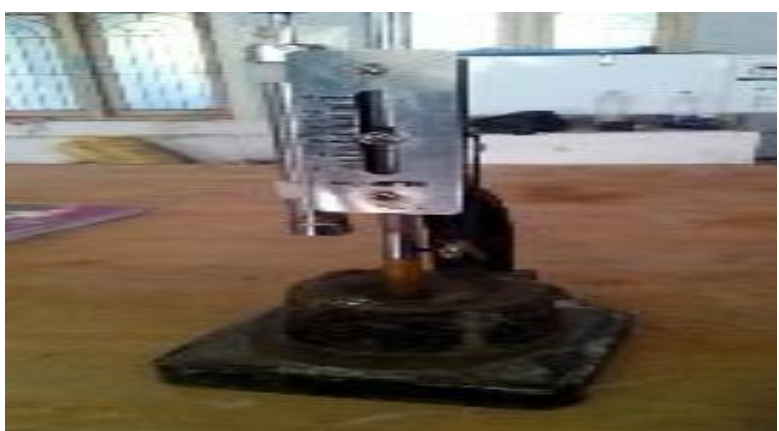

Figure 3.7: Normal Consistancy method

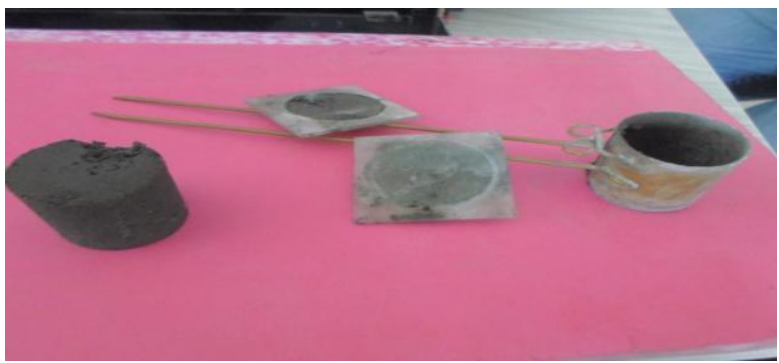

Figure 3.8: Le Chatelier Apparatus 


\section{International Journal of Science and Research (IJSR) \\ ISSN (Online): 2319-7064}

Index Copernicus Value (2013): 6.14 | Impact Factor (2015): 6.391
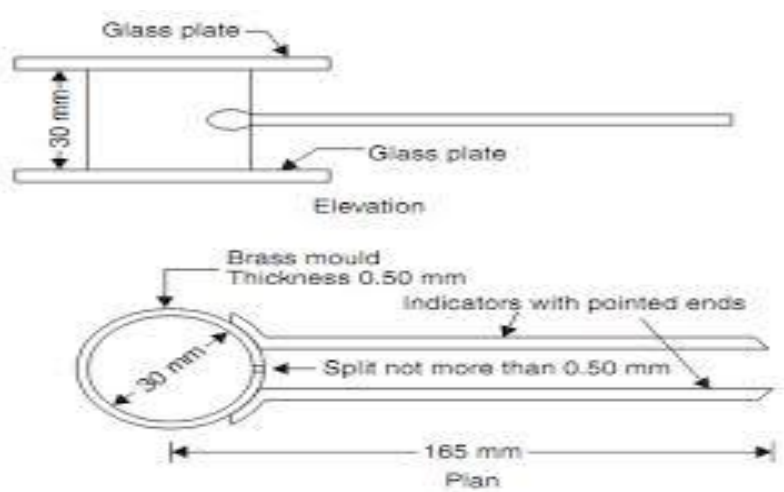

Figure 3.9: Le Chatelier Apparatus with Dimensions

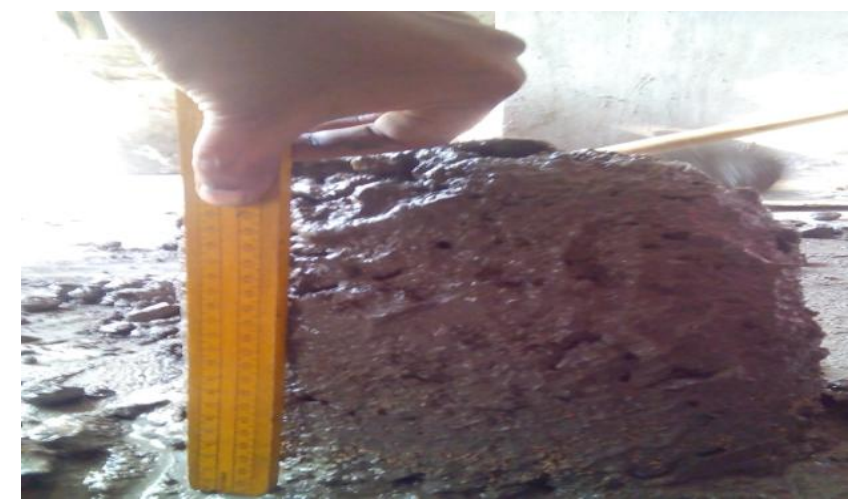

Figure 4.1: Slump Factor of Fresh Concrete

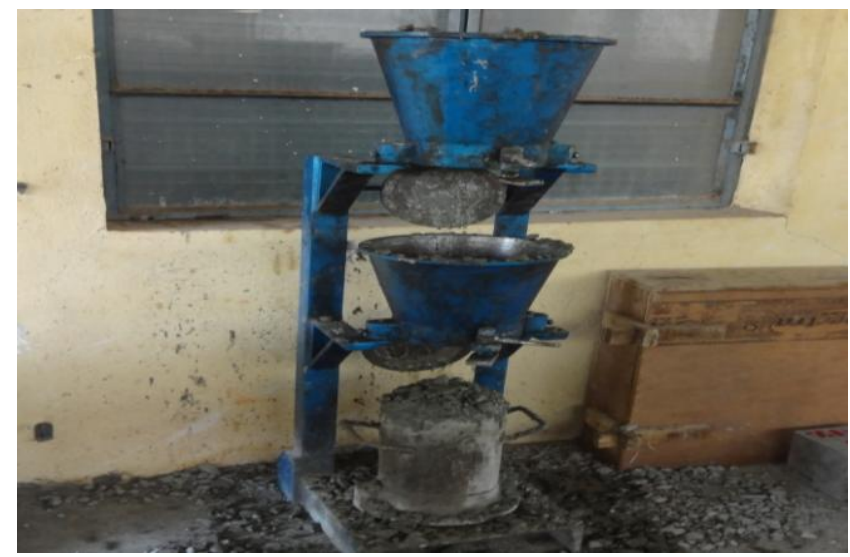

Figure 4.2: Compaction Factor Test

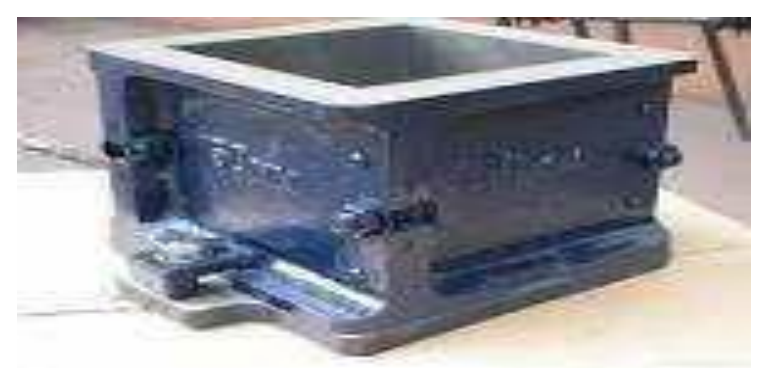

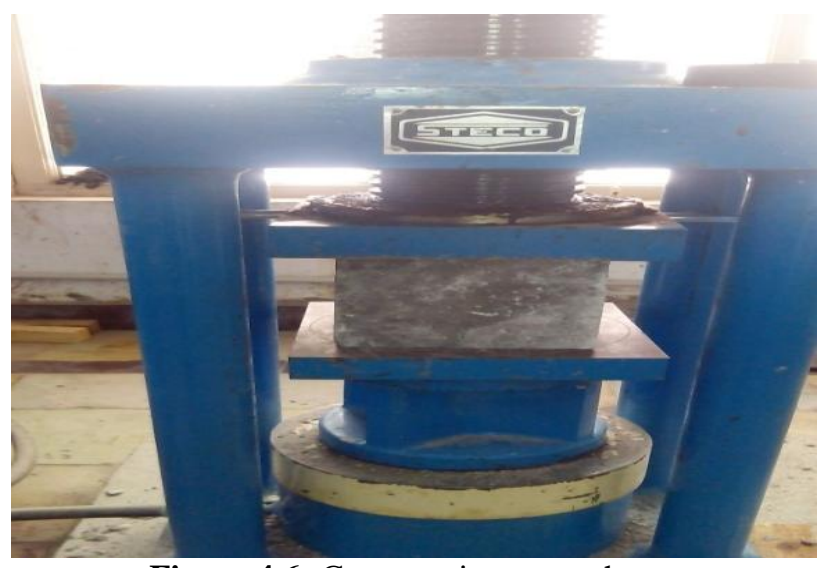

Figure 4.6: Compressive strength test

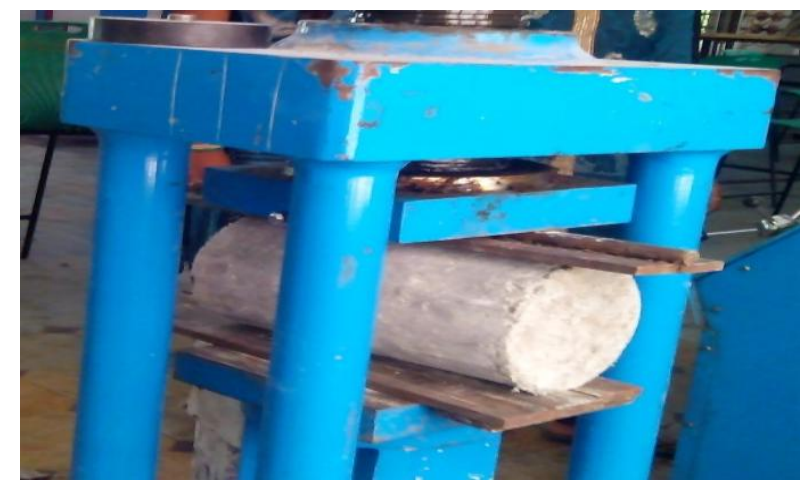

Figure 4.7: Split tensile strength test

\section{Preparation of Concrete}

Production of quality concrete requires meticulous care exercised at every stage of manufacture of concrete. It is interesting that the ingredients of good concrete and bad concrete are the same. If meticulous care is not exercised and good rules are not observed the resultant concrete is going to be of bad quality. With the same material if intense care is taken to exercise control at every stage it will result in good concrete. The various stages of manufacture of concrete are:
(a) Batching
(b) Mixing
(c) Placing
(d) Compaction
(e) Curing

\section{Results}

\subsection{General}

The results of the present investigation are presented both in tabular and graphical forms in order to facilitate the analysis, interpretation of the results is results obtained is based on the current knowledge available in the literature as well as on the nature of results obtained. The significance of the results is assessed with reference to the standards specified by the relevant IS codes.

\subsection{Normal Consistency}

(i)Normal consistency of cement

Volume 5 Issue 6, June 2016 www.ijsr.net 


\section{International Journal of Science and Research (IJSR) \\ ISSN (Online): 2319-7064}

Index Copernicus Value (2013): 6.14 | Impact Factor (2015): 6.391

Table 6.1: Normal consistency of cement

\begin{tabular}{|c|c|c|}
\hline Sl. No & Water percentage (\%) & Depth not penetrated $(\mathrm{mm})$ \\
\hline 1 & 25 & 35 \\
\hline 2 & 27 & 33 \\
\hline 3 & 29 & 24 \\
\hline 4 & 30 & 13 \\
\hline 5 & 31 & 7.4 \\
\hline 6 & 32 & 6 \\
\hline
\end{tabular}

Normal consistency of cement $=32.5 \%$

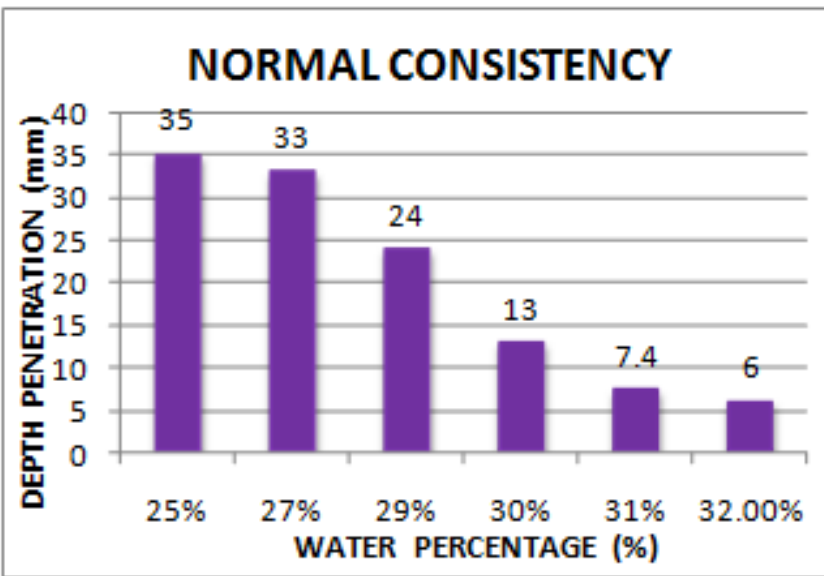

Figure 6.1: Normal Consistency at various water percentages

\subsection{Grain Size Distribution}

Table 6.4.1: Grain size distribution of fine aggregates

\begin{tabular}{|c|c|c|c|c|c|}
\hline S.No & IS Sieve & $\begin{array}{c}\text { Weight } \\
\text { Retained }\end{array}$ & $\begin{array}{c}\text { \%Weight } \\
\text { Retained }\end{array}$ & $\begin{array}{c}\text { Cumulative\% } \\
\text { Weight } \\
\text { Retained }\end{array}$ & \%Passing \\
\hline 1 & $10 \mathrm{~mm}$ & 0 & 0 & 0 & 100 \\
\hline 2 & $4.75 \mathrm{~mm}$ & 15 & 1.5 & 1.5 & 98.5 \\
\hline 3 & $2.36 \mathrm{~mm}$ & 30 & 3.0 & 4.5 & 95.5 \\
\hline 4 & $1.18 \mathrm{~mm}$ & 315 & 31.5 & 36 & 64 \\
\hline 5 & $600 \mu$ & 275 & 27.5 & 63.5 & 36.5 \\
\hline 6 & $300 \mu$ & 210 & 21 & 84.5 & 15.5 \\
\hline 7 & $150 \mu$ & 60 & 6 & 90.5 & 9.5 \\
\hline
\end{tabular}

The grain size distribution of fine aggregates is found taking $1 \mathrm{~kg}$ of sand and is passed through the sieves $(4.75 \mathrm{~m}$, $2.36 \mathrm{~mm}, 1.18 \mathrm{~mm}, 600 \mu, 300 \mu, 150 \mu$, pan lid). Weights of aggregates retained in sieves are noted in above table.

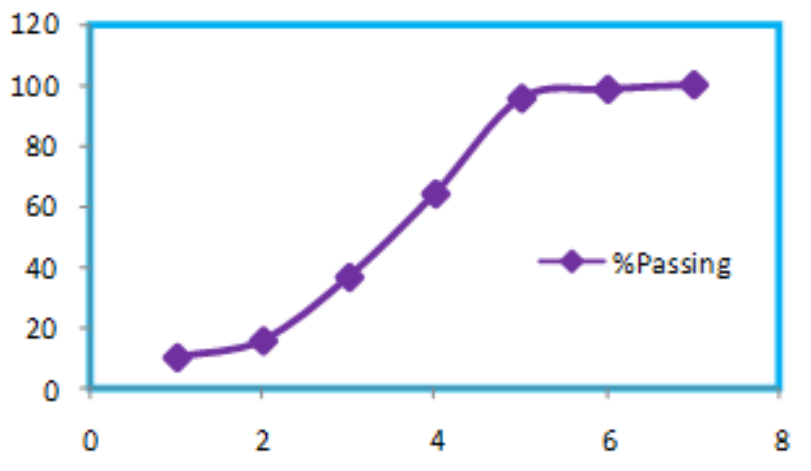

Table 6.4.2: Grain size distribution of coarse aggregates

\begin{tabular}{|c|c|c|c|c|}
\hline S.No & $\begin{array}{c}\text { IS } \\
\text { Sieve }\end{array}$ & $\begin{array}{c}\text { Weight } \\
\text { Retained }\end{array}$ & $\begin{array}{c}\text { \%Weight } \\
\text { Retained }\end{array}$ & $\begin{array}{c}\text { Cumulative \% } \\
\text { weight } \\
\text { Retained }\end{array}$ \\
\hline 1 & $63 \mathrm{~mm}$ & 0 & 0 & 0 \\
\hline 2 & $40 \mathrm{~mm}$ & 0 & 0 & 0 \\
\hline 3 & $20 \mathrm{~mm}$ & 2365 & 47.3 & 0 \\
\hline 4 & $10 \mathrm{~mm}$ & 1625.6 & 32.51 & 47.3 \\
\hline 5 & $4.75 \mathrm{~mm}$ & 984.4 & 19.68 & 79.81 \\
\hline 6 & $2.36 \mathrm{~mm}$ & 24.5 & 0.49 & 99.49 \\
\hline 7 & $1.18 \mathrm{~mm}$ & 0 & 0 & 99.98 \\
\hline 8 & $600 \mu$ & 0 & 0 & 99.98 \\
\hline 9 & $300 \mu$ & 0.3 & 0.006 & 99.986 \\
\hline 10 & $150 \mu$ & 0.2 & 0.004 & 100 \\
\hline
\end{tabular}

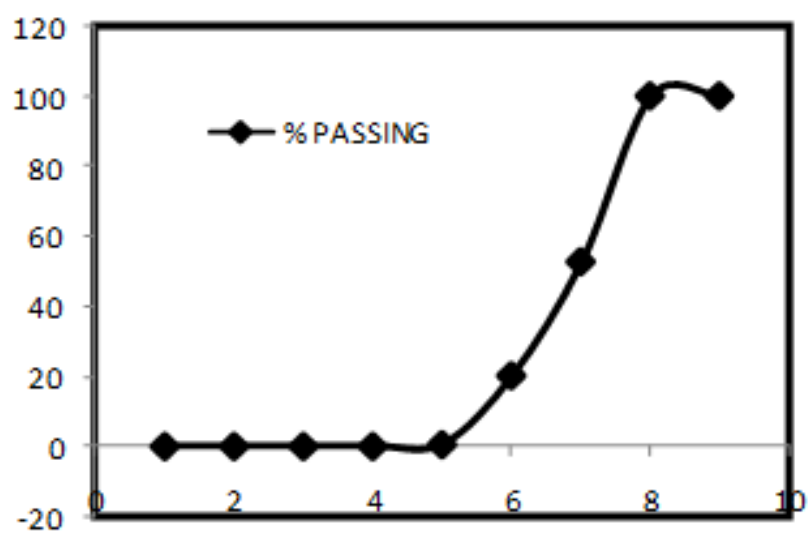

3.4 Variation of compressive strength of different replacement of sand with bottom ash

Table 6.5.1: Variation of Compressive strength (7 days) of different replacements of FA with bottom ash

\begin{tabular}{|c|c|c|c|c|c|c|}
\hline $\begin{array}{l}\mathrm{S} \\
\mathrm{N}\end{array}$ & $\begin{array}{l}\text { Details } \\
\text { (7Days) }\end{array}$ & Cubes & $\begin{array}{l}\text { Load } \\
(\mathrm{N})\end{array}$ & $\begin{array}{c}\text { Area } \\
\left(\mathrm{mm}^{2}\right)\end{array}$ & $\begin{array}{c}\text { Compressive } \\
\text { strength } \\
\left(\mathrm{N} / \mathrm{mm}^{2}\right)\end{array}$ & $\begin{array}{l}\text { Average } \\
\left(\mathrm{N} / \mathrm{mm}^{2}\right)\end{array}$ \\
\hline \multirow[t]{3}{*}{1} & \multirow{3}{*}{$\begin{array}{l}100 \% \mathrm{FA} \\
+0 \% \mathrm{BA}\end{array}$} & 1 & 490000 & 22500 & 21.77 & \multirow[t]{3}{*}{21.17} \\
\hline & & 2 & 470000 & 22500 & 20.88 & \\
\hline & & 3 & 470000 & 22500 & 20.88 & \\
\hline \multirow[t]{3}{*}{2} & \multirow{3}{*}{$\begin{array}{c}90 \% \mathrm{FA} \\
+10 \% \mathrm{BA}\end{array}$} & 1 & 450000 & 22500 & 20 & \multirow[t]{3}{*}{19.25} \\
\hline & & 2 & 420000 & 22500 & 18.66 & \\
\hline & & 3 & 430000 & 22500 & 19.11 & \\
\hline \multirow[t]{3}{*}{3} & \multirow{3}{*}{$\begin{array}{c}80 \% \mathrm{FA} \\
+20 \% \mathrm{BA}\end{array}$} & 1 & 460000 & 22500 & 20.44 & \multirow[t]{3}{*}{20.29} \\
\hline & & 2 & 470000 & 22500 & 20.88 & \\
\hline & & 3 & 440000 & 22500 & 19.55 & \\
\hline \multirow[t]{3}{*}{4} & \multirow{3}{*}{$\begin{array}{r}70 \% \mathrm{FA} \\
+30 \% \mathrm{BA}\end{array}$} & 1 & 460000 & 22500 & 20.44 & \multirow[t]{3}{*}{19.10} \\
\hline & & 2 & 440000 & 22500 & 19.55 & \\
\hline & & 3 & 390000 & 22500 & 17.33 & \\
\hline
\end{tabular}

\section{Compressive strength}

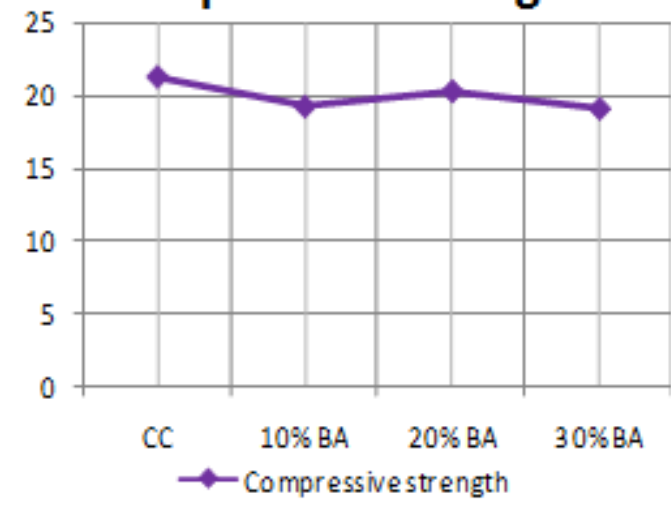

Volume 5 Issue 6, June 2016 www.ijsr.net

Licensed Under Creative Commons Attribution CC BY 


\section{International Journal of Science and Research (IJSR) \\ ISSN (Online): 2319-7064}

Index Copernicus Value (2013): 6.14 | Impact Factor (2015): 6.391

3.4.1 Variation of Compressive strength (7days) for different replacement

3.4.2 Variation of Compressive strength (28 days) of different replacements

Table 6.5.3: Variation of Compressive strength (28 days) of different replacements of FA with bottom ash

\begin{tabular}{|c|c|c|c|c|c|c|}
\hline $\begin{array}{l}\mathrm{S} \\
\mathrm{N}\end{array}$ & $\begin{array}{c}\text { Details(28 } \\
\text { Days) }\end{array}$ & Cubes & $\begin{array}{l}\text { Load } \\
(\mathrm{N})\end{array}$ & $\begin{array}{c}\text { Area } \\
\left(\mathrm{mm}^{2}\right)\end{array}$ & $\begin{array}{c}\text { Compressive } \\
\text { strength } \\
\left(\mathrm{N} / \mathrm{mm}^{2}\right)\end{array}$ & $\begin{array}{l}\text { Average } \\
\left(\mathrm{N} / \mathrm{mm}^{2}\right)\end{array}$ \\
\hline \multirow[t]{3}{*}{1} & \multirow{3}{*}{$\begin{array}{l}100 \% \text { FA } \\
+0 \% \text { BA }\end{array}$} & 1 & 620000 & 22500 & 27.55 & \multirow[t]{3}{*}{28.73} \\
\hline & & 2 & 670000 & 22500 & 29.77 & \\
\hline & & 3 & 650000 & 22500 & 28.88 & \\
\hline \multirow[t]{3}{*}{2} & \multirow{3}{*}{$\begin{array}{r}90 \% \mathrm{FA} \\
+10 \% \mathrm{BA}\end{array}$} & 1 & 640000 & 22500 & 28.44 & \multirow[t]{3}{*}{27.1} \\
\hline & & 2 & 590000 & 22500 & 26.22 & \\
\hline & & 3 & 600000 & 22500 & 26.66 & \\
\hline \multirow[t]{3}{*}{3} & \multirow{3}{*}{$\begin{array}{r}80 \% \mathrm{FA} \\
+20 \% \mathrm{BA}\end{array}$} & 1 & 620000 & 22500 & 27.55 & \multirow[t]{3}{*}{27.7} \\
\hline & & 2 & 610000 & 22500 & 27.11 & \\
\hline & & 3 & 640000 & 22500 & 28.44 & \\
\hline \multirow[t]{3}{*}{4} & \multirow{3}{*}{$\begin{array}{c}70 \% \mathrm{FA} \\
+30 \% \mathrm{BA}\end{array}$} & 1 & 640000 & 22500 & 28.44 & \multirow[t]{3}{*}{28.42} \\
\hline & & 2 & 660000 & 22500 & 29.33 & \\
\hline & & 3 & 620000 & 22500 & 27.55 & \\
\hline
\end{tabular}

\section{Compressive strength}

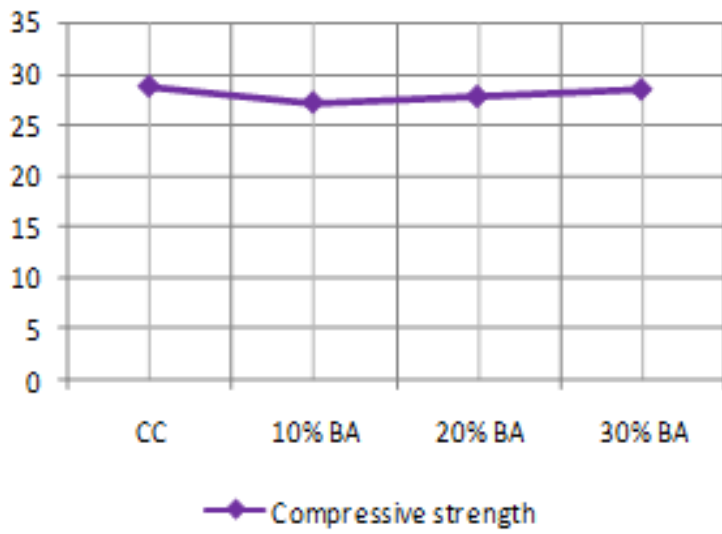

Figure 6.5.3: Variation of Compressive strength (28 days) of different replacement

3.4.3 Variation of Compressive strength (56 days) of different replacements

Table 6.5.4: Variation of Compressive strength (56 days) of different replacements of FA with bottom ash

\begin{tabular}{|c|c|c|c|c|c|c|}
\hline$S N$ & \begin{tabular}{|c|} 
Details \\
( 56 \\
Days)
\end{tabular} & Cubes & $\begin{array}{c}\text { Load } \\
(N)\end{array}$ & $\begin{array}{l}\text { Area } \\
\left(\mathrm{mm}^{2}\right)\end{array}$ & $\begin{array}{c}\text { Compressive } \\
\text { strength } \\
\left(\mathrm{N} / \mathrm{mm}^{2}\right)\end{array}$ & $\begin{array}{l}\text { Average } \\
\left(\mathrm{N} / \mathrm{mm}^{2}\right)\end{array}$ \\
\hline \multirow[t]{3}{*}{1} & \multirow{3}{*}{$\begin{array}{l}100 \% \mathrm{FA} \\
+0 \% \mathrm{BA}\end{array}$} & 1 & 7800000 & 22500 & 34.66 & \multirow[t]{3}{*}{35.25} \\
\hline & & 2 & 8100000 & 22500 & 36.00 & \\
\hline & & 3 & 7900000 & 22500 & 35.11 & \\
\hline \multirow[t]{3}{*}{2} & \multirow{3}{*}{$\begin{array}{c}90 \% \mathrm{FA} \\
+10 \% \\
\mathrm{BA}\end{array}$} & 1 & 7400000 & 22500 & 32.88 & \multirow[t]{3}{*}{33.62} \\
\hline & & 2 & 7700000 & 22500 & 34.22 & \\
\hline & & 3 & 7600000 & 22500 & 33.77 & \\
\hline \multirow[t]{3}{*}{3} & \multirow{3}{*}{$\begin{array}{c}80 \% \mathrm{FA} \\
+20 \% \\
\mathrm{BA}\end{array}$} & 1 & 7600000 & 22500 & 33.77 & \multirow[t]{3}{*}{34.51} \\
\hline & & 2 & 7800000 & 22500 & 34.66 & \\
\hline & & 3 & 7900000 & 22500 & 35.11 & \\
\hline \multirow[t]{3}{*}{4} & \multirow{3}{*}{$\begin{array}{c}70 \% \mathrm{FA} \\
+30 \% \\
\mathrm{BA}\end{array}$} & 1 & 7400000 & 22500 & 32.88 & \multirow[t]{3}{*}{33.92} \\
\hline & & 2 & 770000 & 22500 & 34.22 & \\
\hline & & 3 & 780000 & 22500 & 34.66 & \\
\hline
\end{tabular}

\section{Compressive strength}

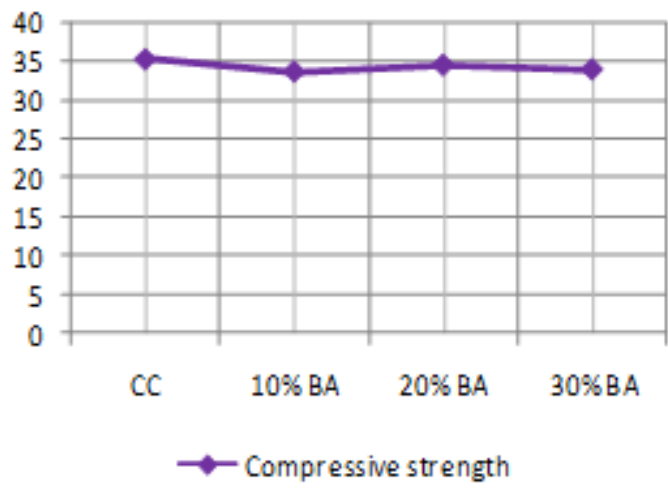

Figure 6.5.4: Variation of Compressive strength (56 days) of different replacement

3.4.4 Variation of Compressive strength (90days) of different replacements

Table 6.5.5: Variation of Compressive strength (90 days) of different replacements of FA with bottom ash

\begin{tabular}{|c|c|c|c|c|c|c|}
\hline SN & \begin{tabular}{|c|} 
Details \\
( 90 \\
Days)
\end{tabular} & Cubes & $\begin{array}{c}\text { Load } \\
(\mathrm{N})\end{array}$ & $\begin{array}{c}\text { Area } \\
\left(\mathrm{mm}^{2}\right)\end{array}$ & $\begin{array}{l}\text { Compressive } \\
\text { strength } \\
\left(\mathrm{N} / \mathrm{mm}^{2}\right)\end{array}$ & $\begin{array}{l}\text { Average } \\
\left(\mathrm{N} / \mathrm{mm}^{2}\right)\end{array}$ \\
\hline \multirow[t]{3}{*}{1} & \multirow{3}{*}{$\begin{array}{c}100 \% \\
\text { FA } \\
+0 \% \mathrm{BA}\end{array}$} & 1 & 870000 & 22500 & 38.66 & \multirow[t]{3}{*}{39.69} \\
\hline & & 2 & 890000 & 22500 & 39.55 & \\
\hline & & 3 & 920000 & 22500 & 40.88 & \\
\hline \multirow[t]{3}{*}{2} & \multirow{3}{*}{$\begin{array}{c}90 \% \text { FA } \\
+10 \% \\
\text { BA }\end{array}$} & 1 & 820000 & 22500 & 36.44 & \multirow[t]{3}{*}{36.59} \\
\hline & & 2 & 840000 & 22500 & 37.33 & \\
\hline & & 3 & 810000 & 22500 & 36 & \\
\hline \multirow[t]{3}{*}{3} & \multirow{3}{*}{$\begin{array}{c}80 \% \text { FA } \\
+20 \% \\
\text { BA }\end{array}$} & 1 & 830000 & 22500 & 36.88 & \multirow[t]{3}{*}{37.03} \\
\hline & & 2 & 840000 & 22500 & 37.33 & \\
\hline & & 3 & 830000 & 22500 & 36.88 & \\
\hline \multirow[t]{3}{*}{4} & \multirow{3}{*}{$\begin{array}{c}70 \% \text { FA } \\
+30 \% \\
\text { BA }\end{array}$} & 1 & 880000 & 22500 & 39.11 & \multirow[t]{3}{*}{39.25} \\
\hline & & 2 & 900000 & 22500 & 40.00 & \\
\hline & & 3 & 870000 & 22500 & 38.66 & \\
\hline
\end{tabular}

\section{Compressive strength}

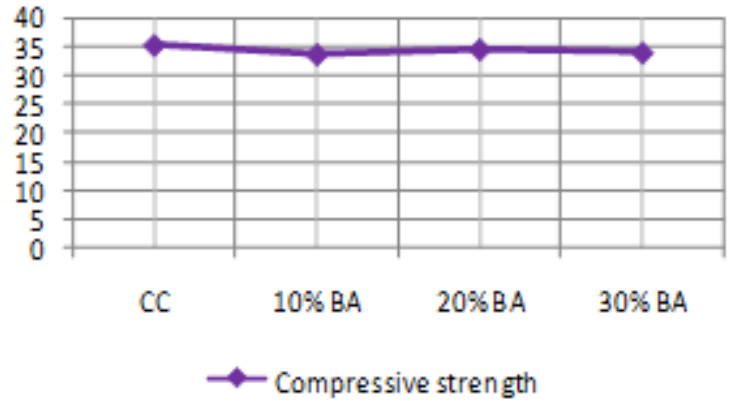

Figure 6.5.5: Variation of Compressive strength (90 days) of different replacement

3.4.5 Variation of Compressive strength containing different replacements

Table 6.6: Compressive strength of concrete containing different replacements of FA with bottom ash

\begin{tabular}{|c|c|c|c|c|c|}
\hline \multirow{2}{*}{ Mix } & \multicolumn{5}{|c|}{ Compressive strength } \\
\cline { 2 - 7 } & 7 days & 14 days & 28 days & 56 days & 90 days \\
\hline $100 \%$ FA+0\% BA & 21.17 & 21.10 & 28.73 & 35.25 & 39.69 \\
\hline $90 \%$ FA+10\% BA & 19.25 & 21.62 & 27.1 & 33.62 & 36.59 \\
\hline $80 \%$ FA+20\% BA & 20.29 & 22.51 & 27.7 & 34.51 & 37.03 \\
\hline $70 \%$ FA+30\% BA & 19.10 & 24.73 & 28.42 & 33.92 & 39.25 \\
\hline
\end{tabular}

Volume 5 Issue 6, June 2016 www.ijsr.net 


\section{International Journal of Science and Research (IJSR) \\ ISSN (Online): 2319-7064}

Index Copernicus Value (2013): 6.14 | Impact Factor (2015): 6.391

\section{Compressive strength}

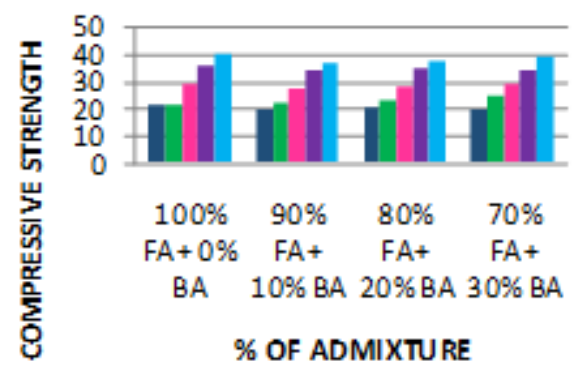

Table 6.8: Compressive strength of concrete containing different replacements of FA with bottom ash using

SUPERPLASTICIZER (4\%)

\begin{tabular}{|c|c|c|c|c|c|}
\hline \multirow{2}{*}{ Mix } & \multicolumn{5}{|c|}{ Compressive strength } \\
\cline { 2 - 6 } & 7 days & 14 days & 28 days & 56 days & 90 days \\
\hline $100 \%$ FA+0\% BA+4\%SP & 22.22 & 21.47 & 33.92 & 36.14 & 37.62 \\
\hline $90 \%$ FA+10\% BA+4\%SP & 20.88 & 21.62 & 37.77 & 42.36 & 48.29 \\
\hline $80 \%$ FA+20\% BA+4\%SP & 22.66 & 22.51 & 43.70 & 43.70 & 45.18 \\
\hline $70 \%$ FA+30\% BA+4\%SP & 20.30 & 24 & 43.14 & 45.62 & 48.14 \\
\hline
\end{tabular}

Figure 6.6: Variation of different replacements of FA with bottom ash

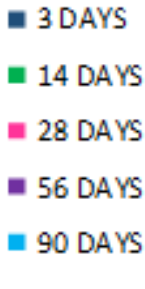

- 3DAYS

28 DAYS

56 DAYS

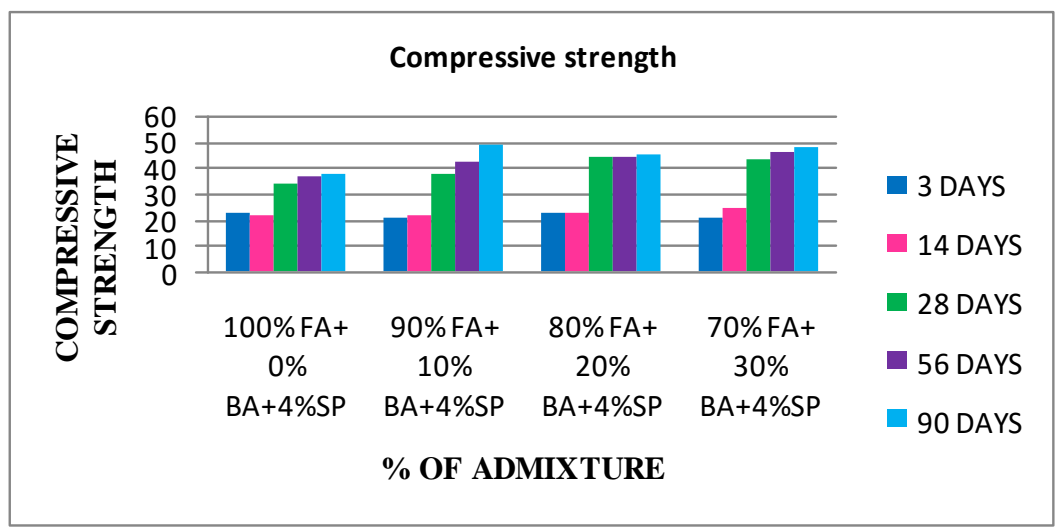

3.4.6 Variation of split tensile strength of different replacement of sand with bottom ash using super plasticizer $(4 \%)$

3.4.7 Variation of Split tensile strength (28 days) of different replacements:-

Table 6.8.2: Variation of Split strength (28 days) of different replacements of FA with bottom ash using SUPER PLASTICIZER (4\%)

\begin{tabular}{|c|c|c|c|c|c|}
\hline $\begin{array}{l}\text { S. } \\
\text { No }\end{array}$ & $\begin{array}{c}\text { Details } \\
\text { (28 Days) }\end{array}$ & Cylinder & $\begin{array}{l}\text { Load } \\
(\mathrm{N})\end{array}$ & $\begin{array}{l}\text { Split tensile } \\
\text { strength } \\
\left(\mathrm{N} / \mathrm{mm}^{2}\right)\end{array}$ & $\begin{array}{c}\text { Avg } \\
\left(\mathrm{N} / \mathrm{m}^{2}\right)\end{array}$ \\
\hline \multirow[t]{2}{*}{1} & \multirow{2}{*}{$\begin{array}{c}100 \% \mathrm{FA} \\
+0 \% \mathrm{BA}+4 \% \mathrm{SP}\end{array}$} & 1 & 300000 & 4.24 & \multirow[t]{2}{*}{4.1} \\
\hline & & 2 & 280000 & 3.96 & \\
\hline \multirow[t]{2}{*}{2} & \multirow{2}{*}{$\begin{array}{c}90 \% \mathrm{FA} \\
+10 \% \mathrm{BA}+4 \% \mathrm{SP} \\
\end{array}$} & 1 & 250000 & 3.53 & \multirow[t]{2}{*}{3.46} \\
\hline & & 2 & 240000 & 3.39 & \\
\hline \multirow[t]{2}{*}{3} & \multirow{2}{*}{$\begin{array}{c}80 \% \mathrm{FA} \\
+20 \% \mathrm{BA}+4 \% \mathrm{SP} \\
\end{array}$} & 1 & 260000 & 3.67 & \multirow[t]{2}{*}{3.67} \\
\hline & & 2 & 260000 & 3.67 & \\
\hline \multirow[t]{2}{*}{4} & \multirow{2}{*}{$\begin{array}{c}70 \% \mathrm{FA} \\
+30 \% \mathrm{BA}+4 \% \mathrm{SP}\end{array}$} & 1 & 280000 & 3.96 & \multirow[t]{2}{*}{3.68} \\
\hline & & 2 & 240000 & 3.39 & \\
\hline
\end{tabular}

\section{Split tensile strength}

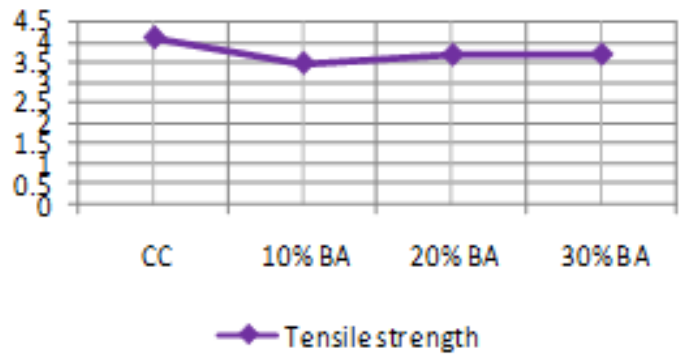

Figure 6.8.2: Variation of Split tensile strength (28days) of different replacement using super plasticizer (4\%)
Table 6.9: Variation of Split strength (28 days) of different replacements of FA with bottom ash using without and with SUPER PLASTICIZER (4\%)

\begin{tabular}{|c|c|c|}
\hline \multirow{2}{*}{ Mix } & \multicolumn{2}{|c|}{ Split Tensile Strength (28 DAYS) } \\
\cline { 2 - 3 } & WITHOUT SP & WITH SP (4\%) \\
\hline $100 \% \mathrm{FA}+0 \% \mathrm{BA}$ & 2.41 & 4.1 \\
\hline $90 \% \mathrm{FA}+10 \% \mathrm{BA}$ & 2.76 & 3.46 \\
\hline $80 \% \mathrm{FA}+20 \% \mathrm{BA}$ & 3.32 & 3.67 \\
\hline $70 \% \mathrm{FA}+30 \% \mathrm{BA}$ & 3.61 & 3.68 \\
\hline
\end{tabular}

\section{Split tensile strength}

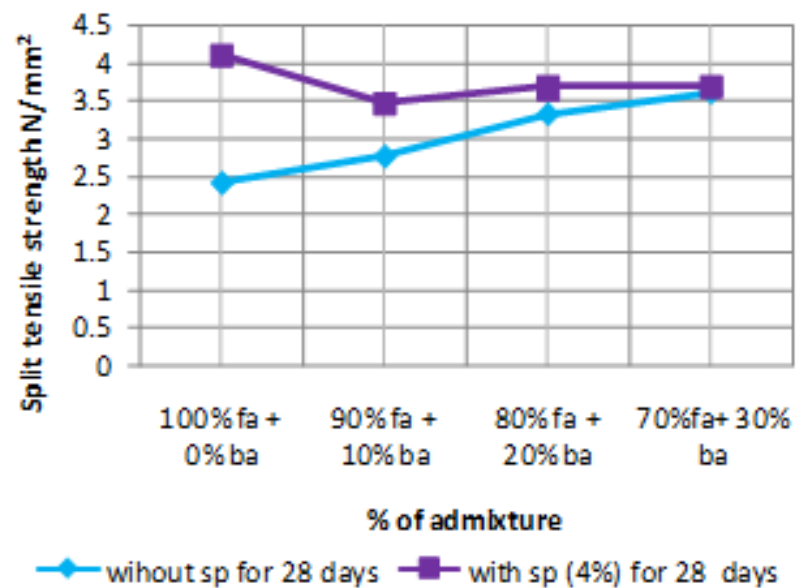

Figure 6.9.1: Variation of Split tensile strength (28days) of different replacement using super plasticizer (4\%)

Volume 5 Issue 6, June 2016 www.ijsr.net

Licensed Under Creative Commons Attribution CC BY 


\section{International Journal of Science and Research (IJSR) \\ ISSN (Online): 2319-7064}

Index Copernicus Value (2013): 6.14 | Impact Factor (2015): 6.391

\subsubsection{Durability Tests}

\subsubsection{Water Absorption Test}

Table 6.10: Water Absorption Test for Different Proportions of Concrete

\begin{tabular}{|c|c|c|c|c|}
\hline S.No & Sample & $\begin{array}{c}\text { Wet } \\
\text { weight } \\
(\mathrm{kg}) \\
(\mathrm{A})\end{array}$ & $\begin{array}{c}\text { Dry } \\
\text { weight } \\
(\mathrm{kg}) \\
(\mathrm{B})\end{array}$ & $\begin{array}{c}\text { Water absorption } \\
\text { in \% } \\
(\mathrm{A}-\mathrm{B} / \mathrm{B}) \times 100\end{array}$ \\
\hline 1 & $100 \% \mathrm{OPC}+0 \% \mathrm{BA}$ & 8.796 & 8.438 & 4.24 \\
\hline 2 & $90 \% \mathrm{OPC}+10 \% \mathrm{BA}$ & 8.764 & 8.193 & 6.96 \\
\hline 3 & $80 \% \mathrm{OPC}+20 \% \mathrm{BA}$ & 8.785 & 8.166 & 7.58 \\
\hline 4 & $70 \% \mathrm{OPC}+30 \% \mathrm{BA}$ & 8.803 & 7.971 & 9.27 \\
\hline
\end{tabular}

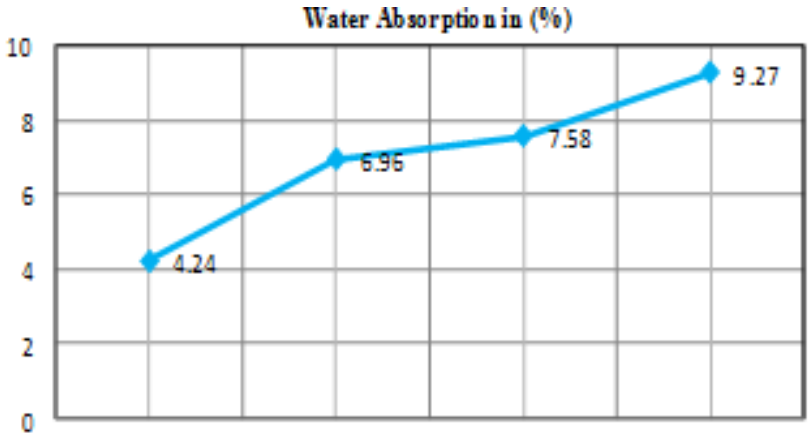

100\%OPC+0\%BA 90\%OPC+10\%BA $80 \% O P C+20 \% B A \quad 70 \% O P C+30 \% B A$ $\rightarrow-$ Water Absorption in (\%)

Figure 6.10: water absorption for different cubes in for 28 days.

Table 6.12: Variation of Water absorption (28 days) of different replacements of FA with BOTTOM ASH using without and with SUPER PLASTICIZER (4\%)

\begin{tabular}{|c|c|c|}
\hline \multirow{2}{*}{ Mix } & \multicolumn{2}{|c|}{ WATER ABSORPTION (28 DAYS) IN \% } \\
\cline { 2 - 3 } & WITHOUT SP & WITH SP $(4 \%)$ \\
\hline $100 \% \mathrm{FA}+0 \% \mathrm{BA}$ & 4.24 & 1.73 \\
\hline $90 \% \mathrm{FA}+10 \% \mathrm{BA}$ & 6.96 & 1.85 \\
\hline $80 \% \mathrm{FA}+20 \% \mathrm{BA}$ & 7.58 & 2.12 \\
\hline $70 \% \mathrm{FA}+30 \% \mathrm{BA}$ & 9.27 & 2.22 \\
\hline
\end{tabular}

Variation of Wa terabso p pixa in $\%$ using without and with super plasticier (28 days)

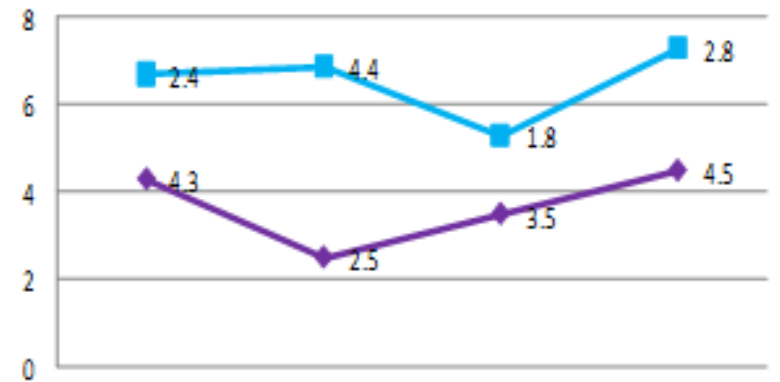

$100 \% F A+0 \%$ BA 90\% FA + 10\% BA 80\% FA + 20\% BA 70\%FA+30\%BA - -without sp - -with sp

Figure 6.12: Variation of Water absorption in \% using without and with super plasticizer (28 days)

\section{Conclusions}

Bottom Ash is used in production of concrete cubes by replacement levels of $10 \%, 20 \%, 30 \%$ by weight of sand (WITHOUT SUPERPLASTICIZER). These cubes were cured and tested for compressive strength for 7 days, 28 days, 56 days, 90 days and results were noted. Based on the experimental investigation conducted the following conclusions are drawn. Different industrial waste materials are used as partial replacement of sand at different levels produced concrete with high strength and acceptable structural grade concrete. Curing conditions adopted in this research produced significant changes in the properties of concretes especially those containing different replacement levels.

- The most interesting result was that bottom ash retards the initial setting time and accelerates the final setting time.

- The soundness of cement was found to be increase after the replacement of bottom ash.

- The compressive strength value and permeability properties of concrete decreases with increasing bottom ash content. As the replacement percentage of bottom ash is increased, more entrapped air occurs and this causes a negative effect on strength and permeability properties.

- High volume bottom ash replacement is not appropriate because of its high water absorption capacity.

- The compressive strength results were (Without Super plasticizer)

$\rightarrow$ At $30 \%$ replacement of bottom ash, the maximum compressive strength for 28 days was $28.42 \mathrm{~N} / \mathrm{mm}^{2}$

$\rightarrow$ At $10 \%$ replacement of bottom ash, the minimum compressive strength for 28 days was $27.71 \mathrm{~N} / \mathrm{mm}^{2}$

- Similarly the compressive strength results were (With Super plasticizer)

$\rightarrow$ At $20 \%$ replacement of bottom ash, the maximum compressive strength for 28 days was $43.70 \mathrm{~N} / \mathrm{mm}^{2}$.

$\rightarrow$ At $0 \%$ replacement of bottom ash that means, the minimum compressive strength for 28 days was 33.92 $\mathrm{N} / \mathrm{mm}^{2}$

- The split tensile strength results were (without super plasticizer)

$\rightarrow$ At $30 \%$ replacement of bottom ash, the maximum tensile strength for 28 days was $3.61 \mathrm{~N} / \mathrm{mm}^{2}$.

$\rightarrow$ At $0 \%$ replacement of bottom ash it means normal concrete, the minimum tensile strength for 28 days was $2.41 \mathrm{~N} / \mathrm{mm}^{2}$.

- Similarly, the split tensile strength results were (with super plasticizer)

$\rightarrow$ At normal concrete, the maximum tensile strength for 28 days was $4.1 \mathrm{~N} / \mathrm{mm}^{2}$.

$\rightarrow$ At $10 \%$ replacement of bottom ash, the minimum tensile strength for 28 days was $3.46 \mathrm{~N} / \mathrm{mm}^{2}$.

- The durability tests named water absorption test and calorimetric chlorination technique results were

$\rightarrow$ The water absorption is high compared to normal cubes. At $10 \%$ of bottom ash (without super plasticizer), the water absorption is $6.8 \%$ are almost equated to normal concrete of $4.24 \%$.

$\rightarrow$ The water absorption is high compared to normal cubes. At $10 \%$ of bottom ash (with super plasticizer), the water absorption is $1.85 \%$ are almost equated to normal concrete of $1.73 \%$.

\section{References}

[1] IS 456-2000 -Code of practice for plain \& reinforced cement concrete.

[2] IS 12269-1987-Specification for OPC 53 grade.

\section{Volume 5 Issue 6, June 2016 www.ijsr.net}




\section{International Journal of Science and Research (IJSR) \\ ISSN (Online): 2319-7064}

Index Copernicus Value (2013): 6.14 | Impact Factor (2015): 6.391

[3] IS 10262-1982-Recommended guide line for concrete mix design.

[4] IS 3812-1981- Specifications for use as pozzolana\& admixture (First Revision).

[5] IS 3812(Part1)-2003-Specifications for pulverized fuel ash for use as cement, cement mortar and concrete (Second Revision).

[6] IS 9103-1999-Concrete admixture Specifications.

[7] IS 516-1965-Method of Test for Strength of Concrete.

[8] IS 383-1970-Specification for coarse aggregate and fine aggregate from natural sources.

[9] IS 650-1966-Specification for standard sand for testing of cement.

Volume 5 Issue 6, June 2016 www.ijsr.net 